\title{
A CHARACTERIZATION OF $H^{2}$ CLASSES ON RANK ONE SYMMETRIC SPACES OF NONCOMPACT TYPE
}

\author{
PATRICIO CIFUENTES
}

(Communicated by J. Marshall Ash)

\begin{abstract}
A characterization of the Hardy class $H^{2}$ on a rank one symmetric space of noncompact type by a Littlewood-Paley type operator defined through the Green potential of the norm square of the invariant gradient.
\end{abstract}

\section{O. INTRODUCTION}

In the present article we characterize $H^{2}$-classes of harmonic functions on rank one symmetric spaces of noncompact type using a Littlewood-Paley type operator that is defined as the Green potential of the square of the gradient. It seems that this type of operator was first suggested by R. Gundy (cf. GetoorSharpe [5] and Meyer [10]) to characterize BMO of $\mathbf{R}_{+}^{n+1}$. The idea of using this operator to characterize $H^{p}$ is due to Debiard (cf. Debiard [4]). The method that we follow here is to show that the Littlewood-Paley operator of harmonic function verifies a $L^{2}$-boundedness if and only if the area integral of that function is in $L^{2}$.

In $\S 1$ we state several facts about symmetric spaces and give the basic definitions. The reader is referred to Helgason [6] and Koranyi [9] for more details. In $\S 2$ we state and prove our results.

\section{Generalities}

Let $\mathbf{X}$ be a rank one symmetric space of noncompact type. Let $G$ be the identity component of the group of isometries of $\mathbf{X}$. Fix a point $\mathbf{o} \in \mathbf{X}$ as the base point for the space and let $K$ be the isotropy subgroup of $G$ at $\mathbf{0}$. The group $G$ is a semisimple Lie group with finite center, and $K$ is a compact subgroup. The space $\mathbf{X}$ can be identified with the homogeneous space $G / K$. The Lie algebras of $G$ and $K$ are denoted as $\mathbf{g}$ and $\mathbf{k}$. Let $\theta$ be the Cartan involution in $\mathbf{g}$ relative to $\mathbf{k}$ and let $\mathbf{p}$ be the subspace of $\mathbf{g}$ with eigenvalue1 relative to $\theta: \mathbf{g}=\mathbf{k} \oplus \mathbf{p}$. Let a be a fixed maximal subalgebra of $\mathbf{p}$, the dimension of $\mathbf{a}$ is the same as the rank of $\mathbf{X}$, in our case it is 1 , therefore

Received by the editors March 1, 1988 and, in revised form, June 15, 1988.

1980 Mathematics Subject Classification (1985 Revision). Primary 22E30, 43A85. 
$\mathbf{a}=\{\alpha H: \alpha \in \mathbf{R}\}$ for fixed $H \in \mathbf{a}$. The homomorphism ad $H$ of $G$, defined as $\operatorname{ad} H(X)=[H, X]$, can have either 3 or 5 eigenvalues, namely $\{0, \pm \lambda\}$ or $\{0, \pm \lambda, \pm 2 \lambda\}$. We fix $H$ such that $\lambda(H)=1$ and denote the eigenspaces as $\mathbf{g}_{0}, \mathbf{g}_{ \pm 1}, \mathbf{g}_{ \pm 2}$. In particular $\mathbf{g}_{-1}=\{X \in \mathbf{g}:[H, X]=-X\}$ and $\mathbf{g}_{-2}=$ $\{X \in \mathbf{g}:[H, X]=-2 X\}$. Let $p$ and $q$ be the dimensions of $\mathbf{g}_{-1}$ and $\mathbf{g}_{-2}$ respectively, then $q$ can only be $0,1,3$ and 7 , and $p$ ranges over an infinite set of positive integers, except when $q=7$, in which case $p=8$.

Let $\overline{\mathbf{n}}=\mathbf{g}_{-1}+\mathbf{g}_{-2}$ and let $\bar{N}$ and $A$ be the analytic subgroups of $G$ with Lie algebras $\overline{\mathbf{n}}$ and a respectively:

$$
A=\{\exp s H: s \in \mathbf{R}\}, \quad \bar{N}=\left\{\exp (X+Y): X \in \mathbf{g}_{-1}, Y \in \mathbf{g}_{-2}\right\} .
$$

The Iwasawa decomposition of $G=\bar{N} A K$ can be used to introduce a coordinate system in $\mathbf{X}$, namely any $\mathbf{x} \in \mathbf{X}$ admits a unique decomposition as $\mathbf{x}=$ $n a \cdot \mathbf{0}$, where $n \in \bar{N}$ and $a \in A$, therefore $\mathbf{x}=\exp (X+Y) \exp s H \cdot \mathbf{0}=(X, Y, s)$, where $X \in \mathbf{R}^{p}, Y \in \mathbf{R}^{q}, s \in \mathbf{R}$. The group $A$ acts on $\bar{N}$ by conjugation: if $n=\exp (X+Y)$ and $s \in \mathbf{R}$ then $n^{s}=\exp (s H) \exp (X+Y) \exp (-s H)=$ $\exp \left(X^{\prime}+Y^{\prime}\right)$, where $X^{\prime}=e^{-s} X$ and $Y^{\prime}=e^{-2 s} Y$. In accordance with this action we introduce a homogeneous gauge defining a metric in $\bar{N}$ : if $n=\exp (X+Y)$ then $|n|=\left(|X|^{4}+16|Y|^{2}\right)^{1 / 4}$. The Haar measure of $\bar{N}$ is the Lebesgue measure inherited from $\overline{\mathbf{n}}$. It will be denoted as $|\cdot|$ or as $d n$. If $b_{r}$ is the gauge ball of radius $r$ and center at $e$ (the unit of $\bar{N}$ ), then $\left|b_{r}\right|=c r^{m}$, where the constant $c$ is just $\left|b_{1}\right|$ and $m=p+2 q$ is the homogeneous dimension of $\bar{N}$. For an example cf. Cifuentes [1].

Let $\nabla$ and $\Delta$ be, respectively, the invariant gradient and the LaplaceBeltrami operator of $\mathbf{X}$. A function $F$ is harmonic in $\mathbf{X}$ if $\Delta F=0$. Bounded harmonic functions in $\mathbf{X}$ are in one-to-one correspondence with $L^{\infty}$ functions in $\bar{N}$. As a matter of fact any bounded harmonic function can be written as a Poisson integral of an $L^{\infty}$ function in a unique form (cf. Knapp-Williamson [8]). Therefore the group $\bar{N}$ plays the role of the boundary of $\mathbf{X}$. We define the admissible region of convergence to the point $n \in \bar{N}$ as the domain

$$
n \cdot \Gamma_{\alpha}=\left\{\left(n^{\prime}, s^{\prime}\right) \in \mathbf{X}:\left|n^{-1} n^{\prime}\right|<\alpha e^{-s}\right\} .
$$

These regions play the same role as nontangential cones in $\mathbf{R}_{+}^{n+1}$ (cf. Korányi [9]). We define the admissible maximal function associated to $F$ as

$$
N F(n)=\sup \left\{\left|F\left(n^{\prime}, s\right)\right|:\left(n^{\prime}, s\right) \in n \cdot \Gamma_{\alpha}\right\}
$$

and the area integral as

$$
A F(n)=\left(\int_{n \cdot \Gamma_{n}}|F(\mathbf{x})|^{2} d \mathbf{x}\right)^{1 / 2},
$$

where $d \mathbf{x}$ is the volumen element of $\mathbf{X}$. Both of this functionals define functions in $\bar{N}$ that belong simultaneously to $L^{p}(\bar{N})$ for $0<p<\infty$ (cf. Cifuentes [2]). This property is used to characterize $H^{p}$ spaces in $\mathbf{X}$. As it is shown in 
[2, Lemma 2 on page 139] the result does not depend on the value of $\alpha$. Without loss of generality we can assume $\alpha=1$ and write $\Gamma$ instead of $\Gamma_{\alpha}$.

\section{A CHARACTERIZATION OF $H^{2}$}

Our aim in the present work is to show that $H^{2}(\mathbf{X})$ can be characterized by means of a new functional defined as

$$
G F(\mathbf{x})=\left(\int_{\mathbf{x}} G(\mathbf{x}, \mathbf{y})|\nabla F(\mathbf{y})|^{2} d \mathbf{y}\right)^{1 / 2},
$$

where $G(\mathbf{x}, \mathbf{y})$ is the Green function of the space $\mathbf{X}$. This result is suggested by the similar one in Debiard [4] in the context of the generalized half-space of $\mathbf{C}^{n}$.

Theorem. Let $F$ be a harmonic function $\mathbf{X}$, then $A F \in L^{2}(\bar{N})$ if and only if $G F(n, s) \in L^{2}(\bar{N})$ uniformly in $s \in \mathbf{R}$ and the resulting norms are equivalent.

Proof. Assume first that $A F \in L^{2}(\bar{N})$, then

$$
\begin{aligned}
\|A F\|_{2}^{2} & =\int_{\bar{N}} \int_{u \cdot \Gamma}|\nabla F(n, s)|^{2} e^{m s} d n d s d u \\
& =\int_{\bar{N}} \int_{-\infty}^{+\infty} \int_{\bar{N}} \chi_{u}(n, s)|\nabla F(n, s)|^{2} e^{m s} d n d s d u,
\end{aligned}
$$

where $\chi_{u}(n, s)$ is the characteristic function of the admissible region $u \cdot \Gamma$, i.e. $\chi_{u}(n, s)=1$ if $(n, s) \in u \cdot \Gamma$, and $\chi_{u}=0$ otherwise. Changing the order of integration we obtain

$$
\begin{aligned}
\|A F\|_{2}^{2} & =\int_{-\infty}^{+\infty} \int_{\bar{N}}|\nabla F(n, s)|^{2} e^{m s} \int_{\bar{N}} \chi_{u}(n, s) d u d n d s \\
& =\int_{-\infty}^{+\infty} \int_{\bar{N}} \alpha e^{-m s}|\nabla F(n, s)|^{2} e^{m s} d n d s \\
& =\alpha \int_{-\infty}^{+\infty} \varphi(s) d s,
\end{aligned}
$$

where $\varphi(s)=\int_{\bar{N}}|\nabla(n, s)|^{2} d n$. Observe that $\|A F\|_{2}^{2}<\infty$ if and only if $\varphi \in L^{1}(\bar{N})$.

Let us study now the functional $G F$.

$$
\begin{aligned}
\int_{\bar{N}} G F(n, s)^{2} d n & =\int_{\bar{N}} \int_{\mathbf{x}} G((n, s), \mathbf{x})|\nabla F(\mathbf{x})|^{2} d \mathbf{x} d n \\
& =\int_{\bar{N}} \int_{-\infty}^{+\infty} \int_{\bar{N}} G((n, s),(u, t))|\nabla F(u, t)|^{2} e^{m t} d u d t d n .
\end{aligned}
$$

Integrate first with respect to $n$ and observe that $\int_{\bar{N}} G((n, s),(u, t)) d n$ does not depend on $u$ due to the invariance of the integral under the action of the 
group $\bar{N}$ :

$$
\begin{aligned}
\int_{\bar{N}} G((n, s),(u, t)) d n & =\int_{\bar{N}} G\left(\left(u^{-1} n, s\right),(e, t)\right) d n \\
& =\int_{\bar{N}} G((n, s),(e, t)) d n \\
& =\int_{\bar{N}} G\left(\left(n^{-t}, s-t\right),(e, 0)\right) d n \\
& =\int_{\bar{N}} G((n, s-t),(e, 0)) e^{-m t} d n,
\end{aligned}
$$

where the last step is just the substitution of $n$ for $n^{-t}$ whose Jacobian is $e^{-m t}$.

Write $\psi(s)=\int_{\bar{N}} G((n, s),(e, 0)) d n$, then

$$
\begin{aligned}
\int_{\bar{N}} G F(n, s)^{2} d n & =\int_{-\infty}^{+\infty} \int_{\bar{N}}|\nabla F(u, t)|^{2} \psi(s-t) d u d t \\
& =\int_{-\infty}^{+\infty} \varphi(t) \psi(s-t) d t=\varphi * \psi(s) .
\end{aligned}
$$

In other words, $G F(n, s)$ is in $L^{2}(\bar{N})$ uniformly in $s$ if and only if $\varphi * \psi \in$ $L^{\infty}(\mathbf{R})$.

We will prove in the following two Lemmas that the function $\psi(s)$, which depends only on $G(\mathbf{x}, \mathbf{y})$ and therefore is intrinsic to $\mathbf{X}$, is equal to $C e^{-m s}$ for positive $s$, and equal to $C$ for negative $s$, where $C$ is a positive constant. It follows that $\varphi \in L^{1}(\mathbf{R})$ if and only if $\varphi * \psi \in L^{\infty}(\mathbf{R})$, proving the Theorem.

Lemma 1. The function $\psi$ is well defined.

Proof. The Green function of the space $\mathbf{X}$ is given by

$$
G(\mathbf{x}, \mathbf{y})=\int_{d(\mathbf{x}, \mathbf{y})}^{\infty} \frac{d \rho}{A_{\rho}},
$$

(cf. Helgason [7, Theorem 5.27 on page 316]), where $d(\mathbf{x}, \mathbf{y})$ denotes the geodesic distance from $\mathbf{x}$ to $\mathbf{y}$ and $A_{\rho}$ is the surface area of the geodesic sphere of radius $\rho: A_{\rho}=c \sinh ^{p} \rho \sinh ^{q} 2 \rho$.

We want to see that $\psi(s)$ is finite for every $s$. The worst case is $s=0$ because then we integrate the Green function on a surface that contains the pole. For any other $s$ the resulting integral is only improper at $\infty$ and can be estimated in terms of $\psi(0)$.

$$
\begin{aligned}
\psi(0) & =\int_{\bar{N}} G((n, 0), \mathbf{0}) d n=\int_{\bar{N}} \int_{d((n, 0), \mathbf{0})}^{\infty} \frac{d \rho}{A_{\rho}} d n \\
& =\int_{0}^{\infty} \frac{d \rho}{A_{\rho}} \int_{\{n: d((n, 0), \mathbf{0})<\rho\}} d n .
\end{aligned}
$$

We need to express the distance $d((n, 0), \mathbf{0})$ in coordinates. Following Helga- 
son [6, Theorem 3.8 in page 414] we have $2 \cosh 2 d((n, 0), \mathbf{0})=1+2 c|X|^{2}+$ $\left(1+c|X|^{2}\right)^{2}+4 c|Y|^{2}$, where $c$ is a constant that depends only on the dimensions $p$ and $q$ of $\overline{\mathbf{n}}$. Then

$$
\begin{aligned}
\int_{0}^{\infty} & \frac{d \rho}{A_{\rho}}|\{n: d((n, 0), \mathbf{0})<\rho\}| \\
& =\int_{0}^{\infty} \frac{d \rho}{A_{\rho}}\left|\left\{c|X|^{2}+c|Y|^{2}+\frac{c^{2}}{2}|X|^{4}<\cosh 2 \rho-1\right\}\right| \\
& =\int_{0}^{\infty} \frac{d \rho}{A_{\rho}} \int_{c|X|^{2}+c|Y|^{2}+\left(c^{2} / 2\right)|X|^{4}<\cosh 2 \rho-1} d X d Y \\
& <\int_{0}^{\infty} \frac{d \rho}{A_{\rho}} \int_{|X|^{2}+c|Y|^{2}<\cosh 2 \rho-1} d X d Y \\
& =C \int_{0}^{\infty} \frac{d \rho}{A_{\rho}}(\cosh 2 \rho-1)^{(p+q) / 2} \\
& =C \int_{0}^{\infty} \frac{(\cosh 2 \rho-1)^{(p+q) / 2}}{\sinh ^{p} \rho \sinh ^{q} 2 \rho} d \rho .
\end{aligned}
$$

Let us study the asymptotic behavior of the integrand as $\rho \rightarrow 0$ and as $\rho \rightarrow \infty$ As $\rho \rightarrow 0$,

$$
\frac{(\cosh 2 \rho-1)^{(p+q) / 2}}{\sinh ^{p} \rho \sinh ^{q} 2 \rho}=O\left(\frac{\left(1+\frac{(2 \rho)^{2}}{2 !}+\frac{(2 \rho)^{4}}{4 !}+\cdots-1\right)^{(p+q) / 2}}{\left(\rho+\frac{\rho^{3}}{3 !}+\cdots\right)^{p}\left(2 \rho+\frac{(2 \rho)^{4}}{3 !}+\cdots\right)^{q}}\right)=O(1),
$$

it is therefore integrable at $\rho=0$. As $\rho \rightarrow \infty$,

$$
\frac{(\cosh 2 \rho-1)^{(p+q) / 2}}{\sinh ^{p} \rho \sinh ^{q} 2 \rho}=O\left(\frac{e^{(p+q) \rho}}{e^{p \rho} e^{2 q \rho}}\right)=O\left(e^{-q p}\right) .
$$

In the three cases that $q>0$ the function is integrable also at $\infty$.

In the case $q=0$ we have to sharpen our calculations as $\rho \rightarrow \infty$ :

$$
\begin{aligned}
\int_{1+2 c|X|^{2}+\left(c^{2} / 2\right)|X|^{4}<\cosh 2 \rho} d X & =\int_{\left(1+(c / 2)|X|^{2}\right)^{2}<\cosh ^{2} \rho} d X \\
& =\int_{|X|^{2}<(\cosh \rho-1) /(c / 2)} d X \\
& =C\left(\frac{\cosh \rho-1}{c / 2}\right)^{p / 2} \\
& =O\left(e^{-(p / 2) \rho}\right),
\end{aligned}
$$

and therefore is also integrable.

Lemma 2. The function $\psi(s)$ is a solution of the differential equation $\psi^{\prime}+$ $m \psi^{\prime \prime}=0$ for $s \neq 0$. Furthermore, $\psi(s)=C e^{-m s}$ for $s>0$, and $\psi(s)=C$ for $s<0$.

Proof. We will prove first that $\psi(s)$ is a $C^{2}$-function for $s \neq 0$. It will be enough to show that $(\partial / \partial s) G((n, s), 0)$ and $\left(\partial^{2} / \partial s^{2}\right) G((n, s), 0)$ are uniformly integrable in $\bar{N}$ for every compact interval of $s$ that does not contain 
0 . Considering that $G$ is harmonic we can use Schauder interior estimates (cf. Courant and Hilbert [3, page 335] and Cifuentes [1, Lemma 9 on Page 144]) to obtain

$$
\left|\frac{\partial}{\partial s} G(\mathbf{x}, 0)\right| \leq C_{r} \sup _{\mathbf{x}^{\prime} \in B_{r}(x)}\left|G\left(\mathbf{x}^{\prime}, 0\right)\right|
$$

where, for a given compact interval in $s$ not containing 0 , we choose $r$ small enough for $B_{r}(\mathbf{x})$ not to touch $s=0$. Then

$$
\int_{\bar{N}} \frac{\partial}{\partial s} G((n, s), 0) d n \leq C_{r} \int_{\bar{N}} \sup _{B_{r}(n, s)}\left|G\left(\left(n^{\prime}, s^{\prime}\right), 0\right)\right| d n .
$$

We only need to worry about the uniform convergence of this integral at infinity; therefore we only consider the part of the integral where $d((n, s), 0)>|2 s|$; then

$$
\sup _{B_{r}(n, s)}\left|G\left(\left(n^{\prime}, s^{\prime}\right), 0\right)\right| \leq \sup _{B_{r}(n, s)} \int_{d\left(n^{\prime}, s^{\prime}\right)}^{\infty} \frac{d \rho}{A_{\rho}} \leq \int_{d(n, s) / 2}^{\infty} \frac{d \rho}{A_{\rho}},
$$

and this integral gives a function that is integrable in $n$ uniformly on compact intervals of $s$, as shown in Lemma 1 for a similar integral.

In the same way we can deal with $\partial^{2} G / \partial s^{2}$ and we have that $\psi(s)$ is a $C^{2}$ function and we can differentiate under the integral sign. Observe that $G(n, s)$ vanishes at infinity, therefore the transversal part of the Laplace-Beltrami operator (i.e. the component in the direction of $\bar{N}$ ) also commutes with the integral sign in $\int_{\bar{N}} G((n, s), 0) d n$ and $\psi(s)$ verifies the differential equation $\psi^{\prime \prime}(s)+m \psi^{\prime}(s)=0$. This, together with the fact that $\lim _{s \rightarrow \infty} \psi(s)=0$, implies that $\psi(s)=C e^{-m s}$ for $0<s<\infty$. To obtain $\psi(s)$ for $-\infty<s<0$ we observe that $\psi(-s)=e^{m s} \psi(s)$.

\section{REFERENCES}

1. P. Cifuentes, $H^{p}$-classes on rank one symmetric spaces of noncompact type. I, Nontangential and probabilistic maximal functions, Trans. Amer. Math. Soc. 294 (1986), 133-149.

2. _ $H^{p}$-classes on rank one symmetric spaces of noncompact type. II, Nontangential maximal function and area integral, Bull. Sci. Math. 108 (1984), 355-371.

3. R. Courant and D. Hilbert, Methods of mathematical Physics, vol. II, Interscience, New York, 1962.

4. A. Debiard, Espaces $H^{p}$ au dessus de l'espace hermitien hyperbolique de $\mathbf{C}^{n}(n>1)$, II, J. Funct. Anal. 40 (1981), 185-265.

5. R. K. Getoor and M. I. Sharpe, Conformal martingales, Invent. Math. 16 (1972), 271-308.

6. S. Helgason, Differential geometry, Lie groups and symmetric spaces, Academic Press, New York, 1978.

7. ___ Groups and geometric analysis, Academic Press, New York, 1984.

8. A. W. Knapp and R. E. Williamson, Poisson integrals and semisimple Lie groups, J. Analyse Math. 24 (1972), 53-76. 
9. A. Korányi, Harmonic functions on symmetric spaces, Symmetric Spaces (W. Boothby and G. Weiss, eds.), Marcel Dekker, New York, 1972, 379-412.

10. P. A. Meyer, Le dual de $H^{1}\left(\mathbf{R}^{n}\right)$ : démonstration probabiliste, Lecture Notes in Math. vol. 581, Springer-Verlag, Berlin, New York, 1977 pp. 132-195.

Department of Mathematics, Texas A\&M University, College Station, Texas 77843

Current address: Facultat de Matematiques, Universitat de Barcelona, Gran Via 585, 08071 Barcelona, Spain 